\title{
Where there is no overlap, there is a gap
}

\author{
Nicholas Di Paola ${ }^{[1]}$ and Paolo Marinho de Andrade Zanotto ${ }^{[1]}$
}

[1]. Laboratório de Evolução Molecular e Bioinformática, Departamento de Microbiologia, Instituto de Ciências Biomédicas II, Universidade de São Paulo, São Paulo, SP.

Over the past 60 years, Brazil has seen extensive ruralurban migration, leading to human settlements encroaching on tropical ecosystems. Persistent human interaction with vectors from diverse tropical environments has led to the emergence of zoonotic pathogens varying in pathogenicity and infectivity. Over time, zoonotic viruses adapt to humans, eventually leading to secondary transmission in some cases. However, most zoonotic pathogens result in asymptomatic and/ or dead-end cases, rarely leading to sustained human-human transmission. In very rare cases, a single transmission can explode into an outbreak with sustained networks of humanhuman transmission. This is the current situation in West African nations, initiated by a single emergence of the Zaire Ebola virus (EBOVZ) in Guéckédou in Guinea's eastern rainforest region ${ }^{1}$. Although EBOVZ is a known virus, this was the first record of a case in this region, surprising unprepared health authorities with no experience of encountering Ebola viruses. Owing to ideal conditions, the $25^{\text {th }}$ Ebola virus outbreak quickly spread to the neighboring countries, Sierra Leone and Liberia. As of December 2, 2014, the Centers for Disease Control (CDC) and World Health Organization (WHO) had conjunctly reported 16,933 cases and 6,002 deaths. Critically, the virus itself is not necessarily the main factor explaining the current high EBOVZ case count, as it is behaving similarly to the ones isolated during the first fully recorded outbreak of EBOVZ, isolated in 1976. This time, the virus is not experiencing restrictions with respect to the availability of susceptible hosts, as it shows high transmission in densely populated metropolitan settings. Accordingly, the basic reproductive rate $(R)$ has been estimated to be around 2.56 in areas with the highest transmission rates, which is comparable to fomite-transmitted viruses such as small pox and influenza ${ }^{2}$. Indeed, as we cannot predict the success of a pathogen or the time or location of emergence, we should focus on what we can control, i.e., setting up efficient health systems to report and isolate cases and contacts and maximizing surveillance efforts and resources to prevent future outbreaks. The arrival of all dengue virus serotypes (DENV-1, $-2,-3$, and -4),

\footnotetext{
Address to: Dr. Paolo Marinho de Andrade Zanotto. LEMB/ICB II/USP. Av. Prof. Lineu Prestes 1374, 05508-000 São Paulo, SP, Brasil.

Phone: 5511 3091-8453; Fax: 5511 3091-7354

e-mail: pzanotto@usp.br

Received 3 December 2014

Accepted 9 December 2014
}

West Nile virus, and more recently, the Chikungunya virus (CHIKV) in the Americas is a reminder that even with a strong healthcare foundation, a novel emerging or re-emerging virus can cause morbidity and mortality in major metropolitan settings if it is not identified and reported quickly.

A recent review in Revista da Sociedade Brasileira de Medicina Tropical/Journal of the Brazilian Society of Tropical Medicine discussed the introduction of the Mayaro virus (MAYV) and more recently, CHIKV in Brazil ${ }^{3}$. Both have the potential to cause increased morbidity and mortality in Brazil because of arbovirus infections, and therefore, require our attention.

Originally isolated in the 1950s, CHIKV has migrated through the Caribbean and Americas since then, with imported cases reported in Brazil since 2010. Autochthonous transmission was first recorded in September 2014 in the State of Amapá. CHIKV has already experienced sustained transmission in the States of Bahia and Minas Gerais and may have spread widely already. Both competent Aedes aegypti and Aedes albopictus mosquito vectors of CHIKV in an urban cycle, and they are widespread across Brazil. Moreover, the three genotypes of CHIKV can be transmitted efficiently by all 35 strains of Aedes aegypti and Aedes albopictus, justifying the suggestion that outbreaks in other states are inevitable ${ }^{3}$.

Mayaro virus is another pathogen responsible for sporadic cases in Brazil, mainly in the Amazonian region. With MAYV residing in primate reservoirs, Haemagogus and Aedes aegypti mosquitos initiate transmission via a pseudo-sylvatic cycle, similar to yellow fever ${ }^{3}$. Although there have been no large outbreaks, health authorities should preemptively prepare for a re-emergence in unexpected localities.

Symptoms of CHIKV and MAYV are similar to those of other common infectious agents, such as the dengue virus (DENV), and less common agents, such as Rocio, Oropouche, Saint Louis encephalitis, and yellow fever viruses ${ }^{4}$. Importantly, the availability of rapid and efficient assays precisely identifying viral agents is crucial to diagnose patients and analyze epidemiological data. In addition, for medical personnel to follow proper treatment protocols, diagnostics should be readily available, accurate, sensitive, and economical to differentiate between these agents without a coincidental assumption (i.e., during a dengue outbreak, patients with similar symptoms may be infected by other arboviruses but not subjected to formal serological assay). Interestingly, this mirrors initial diagnostic scenarios in Guinea. According to the CDC, in the early stages 
of Ebola virus (EBOV) infection, patients could present with fever, severe headache, fatigue, muscle pain, vomiting, diarrhea, abdominal pain, or unexplained hemorrhaging. In West Africa, this symptom collection could indicate a number of differential diagnoses including malaria, typhoid fever, leptospirosis, rickettsiosis, African trypanosomiasis, Lassa fever, cholera, Marburg virus, and Crimean-Congo hemorrhagic fever ${ }^{5}$. Therefore, in the early stages of an outbreak, lack of positive confirmatory serological tests could delay health authorities' responses, depending on the disease severity. Logically, health structure, resources, and sociological qualities differ between West African nations and the Americas. However, we must continuously remind ourselves that a similar scenario can occur in Brazil. Are Brazil's diagnostic and response capabilities prepared for the emergence of a novel pathogen?

It has previously been argued that many arbovirus infections have been neglected in Brazil, with few epidemiological studies conducted to understand their distribution. For instance, dengue fever is difficult to distinguish from other acute febrile illnesses, which emphasizes the importance of a differential diagnosis ${ }^{4-6}$. Indeed, most diagnoses in the country are made on clinical and epidemiological grounds. In 2014 (as of November 2), 547,612 clinical cases of dengue fever were reported, but 8,423 (1.54\%) were laboratory confirmed ${ }^{7,8}$. These numbers do not represent the countless mildly symptomatic or nonspecific febrile cases. Therefore, the true rates of infection and transmission are underestimated, and the possibility of infection from other pathogens is ignored. With respect to the CDC Ebola case definition, DENV, CHIKV, and MAYV could cause overlapping symptoms with the above diagnoses, supporting the need for broad-spectrum, sensitive, and economic diagnostic assays to identify infectious agents without concerns regarding cross-reaction of closely related agents. This does not imply that an Ebola outbreak is a given in Brazil but exemplifies a complication that could arise in such a scenario.

The emergence of a novel pathogen should always be preconsidered. Moreover, if it has the potential for establishing itself within a human transmission network, having diagnostic tools to confirm its presence and prompt notification of national health authorities should be priorities. Depending on the pathogen's transmissibility, contact tracing and quarantine should be established rapidly. Although EBOV is not novel in the sense of being unknown, it was novel in the locality of its zoonotic emergence in West Africa. Once a country undergoes a zoonoticrelated emergence event leading to morbidity and mortality, the aftermath leads to preemptive measures to ensure that the scale of the next outbreak is significantly diminished. Continuous sentinel and active surveillance are costly and time consuming from a resource perspective. However, considering resource limitations, early diagnostic efforts and surveillance of emerging pathogens are the best ways to reduce mortality, morbidity, and the enormous health care costs associated with epidemics ${ }^{9}$.

Brazil has thousands of communities surrounded by tropical ecosystems teeming with potentially pathogenic agents. Are there countries with limited resources whose healthcare system have successfuly prevented large outbreaks efficiently and strategically, from which we can learn? Uganda could be a good model; similar in respect to climate and ecological diversity, also limited in resources disposed towards surveillance and epidemic management. Uganda presented a model system of emerging infectious disease control, demonstrating from previous EBOV outbreaks that continuous surveillance, that dedicated teams, devoted to contact tracing, case isolation, and cooperating with local communities and bordering countries, successfully limits case counts and spread ${ }^{10}$. Brazil and other nations could learn from Uganda's experience, applying the approach to CHIKV, MAYV, DENV, and other pathogens. Although our ability to diagnose, treat, trace, and contain pathogen outbreaks has improved over the last decade, it has not been sufficient to prevent large outbreaks of new serotypes of dengue, and there is no indication that CHIKV will be halted. In any case, one has to conceive that what is ongoing in West Africa could happen in Brazil. Where there is no overlap, there is a gap; therefore, we should establish much-needed constructive interactions between governments, academia, and the private sector to continue searching for novel methods of diagnostics, improve surveillance, strategize, and maximize resources to prepare for any future emergence. The question is not one of whether an emergence will occur but when and whether we will be ready.

\section{CONFLICT OF INTEREST}

The authors declare that there is no conflict of interest.

\section{REFERENCES}

1. Gatherer D. The 2014 Ebola virus disease outbreak in west Africa. J Gen Virol 2014; 95 (Pt 8):1619-1624.

2. Althaus CL. Estimating the Reproduction Number of Ebola Virus (EBOV) During the 2014 Outbreak in West Africa. PLoS Currents Outbreaks 2014; doi: 10.1371/currents.outbreaks.91afb5e0f279e7f2 9e7056095255b288. Abstract

3. Figueiredo MLG, Figueiredo LTM. Emerging alphaviruses in the Americas: Chikungunya and Mayaro. Rev Soc Bras Med Trop 2014; 47 : 677-683.

4. Figueiredo LTM. Saint Louis encephalitis virus and other arboviruses in the differential diagnosis for dengue. Rev Soc Bras Med Trop 2014; 47:541-542.

5. West TE, von Saint André-von Arnim A. Clinical Presentation and Management of Severe Ebola Virus Disease. Ann Am Thorac Soc 2014; 11:1341-1350.

6. Messina JP, Brady OJ, Scott TW, Zou C, Pigott DM, Duda KA, et al. Global spread of dengue virus types: mapping the 70 year history. Trends Microbiol 2014; 22:138-146.

7. Teixeira MG, Siqueira JB, Ferreira GLC, Bricks L, Joint G. Epidemiological trends of dengue disease in Brazil (2000-2010): a systematic literature search and analysis. PLoS Negl Trop Dis 2013; 7:e2520.

8. Pan American Health Organization. Number of Reported Cases of Dengue and Andean Figures for 2014 (to week noted by each country). [Internet]. [Updated 2014 November 2]; [Cited 2014 December 3]. Available at: http://www.paho.org/.

9. Daszak P, Lipkin WI. The search for meaning in virus discovery. Curr Opin Virol 2011; 1: 620-623.

10. Mbonye AK, Wamala JF, Nanyunja M, Opio A, Makumbi I, Aceng JR. Ebola viral hemorrhagic disease outbreak in west Africa-lessons from Uganda. Afr Health Sci 2014; 14:495-501. 